\title{
DESENVOLVIMENTO DE UM PROTÓTIPO VEICULAR MONOPOSTO DE ALTA EFICIÊNCIA ENERGÉTICA, COM MOTORIZAÇÃO A COMBUSTÃO INTERNA
}

\author{
Gleydson Hiago Sousa Oliveira ${ }^{1}$, Keyll Carlos Ribeiro Martins ${ }^{1}$, Lucas Pablo Silva Brandão \\ Neves $^{1}$, Anthony Soares de Alencar ${ }^{1}$, Kaio Lourenço Teixeira Barbosa ${ }^{1}$, Evandro Martins \\ Araújo Filho ${ }^{1}$, Kamyla Castro Rodrigues ${ }^{1}$ \\ ${ }^{1}$ Instituto Federal de Educação, Ciência e Tecnologia do Maranhão Campus Monte Castelo, \\ São Luís, Maranhão, Brasil \\ E-mails: gleydson.sousa3@gmail.com, kmartins@ifma.edu.br, lucaspablosbn@ hotmail.com, \\ thony_ma@hotmail.com, kaiobrbs@gmail.com, evandro2.0@ hotmail.com, \\ k_mylacastro@yahoo.com.br
}

\section{RESUMO}

Atualmente um dos temas mais abordados são o aquecimento global e o efeito estufa, fenômenos causados principalmente pelo uso de combustíveis fósseis. A maioria dos motores a combustão utilizam esses tipos de combustíveis, contribuindo assim para o aumento desses fenômenos. Desta forma há a necessidade de investir cada vez mais em tecnologias que diminuam a emissão dos gases estufa, diminuir a poluição atmosférica, melhorando assim a qualidade de vida da população local, além de conservar um recurso natural não renovável. Por apresentarem características únicas se comparados a outros tipos se propulsão, os motores a combustão interna, ainda são largamente utilizados, e ainda lideram as vendas no mercado mundial. O projeto propõe-se no desenvolvimento de um veículo para um único ocupante (monoposto) que priorize o baixo consumo específico de combustível e a baixa emissão de poluentes, através do estudo de cada sistema que contribuem para este objetivo, como: motorização, sistema de alimentação de combustível e transmissão. A utilização de novas tecnologias e inovações nestes componentes diminui drasticamente os números de gases estufa, particulados entre outros elementos nocivos à saúde humana provenientes do uso de combustíveis fósseis.

\section{INTRODUÇÃO}

Com aumento da temperatura global, uma das principais preocupações tem sido a diminuição do consumo de combustíveis fósseis, que são um dos causadores do efeito estufa, além do grande aumento da poluição atmosférica nos grandes centros urbanos. O número de veículos vem aumentando gradativamente fazendo-se necessário a criação de tecnologias que visam diminuir esta emissão de poluentes. Além disto, foi necessária a criação de legislações com os programas de controle de emissões como o Programa de Controle de Poluição do ar por Veículos automotores (PROCONVE), criado em 06 de maio de 1986, a Resolução no 18 do CONAMA, no Brasil, que têm se tornado cada vez mais rigorosa; desta forma a indústria automobilística tem investido em novas tecnologias para otimização do sistema de injeção de combustível, privilegiando o menor consumo de combustível e os baixos índices de emissões 
de gases nocivos ao meio ambiente. Surgiram ainda várias opções no mercado mundial entre elas os veículos elétricos, porém os veículos a combustão interna ainda são a grande maioria, por possuírem características únicas como uma ampla faixa de torque, robustez, e baixo custo de manutenção se comparado a outros tipos de propulsão.

Podemos destacar como forma mais visível de poluição do ar, os resíduos provenientes da combustão em veículos automotores, que são lançados diretamente no ar que respiramos, formada principalmente por gases e material particulado (fuligem). Segundo Berna [5], dentre os poluentes gasosos emitidos por motores a combustão interna, alguns estão relacionados como os mais impactantes a saúde, tais como: óxidos de carbono ( $\mathrm{CO}$ e $\mathrm{CO} 2)$, óxidos sulfúricos (SOx), óxidos de nitrogênio (NOx), hidrocarbonetos aromáticos (HA). Esses gases relacionam-se diretamente dificuldade de oxigenação dos tecidos, o aumento de doenças respiratórias desde inflamações (traqueies e bronquites crônicas) até enfisema pulmonar e broncopneumonias químicas ou infecciosas, além de consequências diretas e indiretas do aumento da temperatura global.

Dentro deste contexto atual, a aplicação e aperfeiçoamento de novas tecnologias como sistemas eletrônicos de gerenciamento de energia e alimentação de combustível, a aplicação de materiais mais leves e resistentes na fabricação dos veículos, sistemas que aproveitem melhor o trabalho gerado pelo propulsor, tem se tornado uma resposta à demanda de mercado e aos órgãos reguladores de emissões.

Desta forma o projeto aqui proposto visa o desenvolvimento de um veículo de alta eficiência energética, avaliando os componentes básico de um veículo automotor, visando proporcionar um melhor rendimento geral, destacando-se seu menor consumo de combustível e menores emissões de poluentes, através do desenvolvimento de novas estratégias de controle energia, proporcionando desta forma reduções significativas nos índices dos gases tóxicos. Este trabalho tem como principal aplicabilidade a preservação de um bem finito e cada vez mais escasso no planeta, e ainda uma melhora na qualidade de vida da população em geral.

\section{INFORMAÇÕES GERAIS}

Durante a realização do projeto proposto, foi realizado um amplo estudo de teorias já estabelecidas, em revistas, artigos, livros e periódicos; relacionados a sistemas veiculares, eletroeletrônica automotiva e sistemas de alimentação de combustível. $\mathrm{O}$ estudo destes temas relacionados a pesquisa são de fundamental importância para a identificação e análise dos processos e variáveis que interferem no funcionamento do modelo final.

Após a conclusão da fase inicial do projeto, foram iniciados os estudos e testes para a definição dos componentes a serem utilizados para o desenvolvimento do veículo proposto, dividindo-se principalmente em estudo da motorização a ser utilizada, estrutura do veículo (chassis), transmissão e sistemas auxiliares (direção, frenagem, segurança...). Os artigos deverão ser enviados em formato digitalizado (doc ou pdf sem senha) com texto completo. $\mathrm{O}$ idioma para artigos submetidos ao SIMEA 2017 é o Português, Inglês ou ambos. A AEA não se responsabiliza por trabalhos que percam a qualidade dos gráficos, caso os mesmos não tenham seguido as normas aqui estipuladas. Além disso, nenhuma revisão dos textos será feita, sendo os mesmos de inteira responsabilidade de seus autores.

\subsection{Motor utilizado}

O propulsor de pequeno porte utilizado como propulsão do veículo desenvolvido está alocado no Laboratório de Mecânica Automotiva - IFMA Monte Castelo, e possui as seguintes características: 
Tabela 1: Dados do propulsor utilizado

\begin{tabular}{|c|c|}
\hline Motor: & $\begin{array}{c}\text { Monocilíndrico, 4 tempos, } \\
\text { Ciclo Otto, OHC }\end{array}$ \\
\hline- Cilindrada & $35 \mathrm{~cm}^{3}$ \\
\hline- Potência nominal & $1,4 \mathrm{cv}$ a $6.000 \mathrm{rpm}$ \\
\hline- Torque máximo & $1,5 \mathrm{~N} . \mathrm{m}$ a $5.000 \mathrm{rpm}$ \\
\hline- Ano de produção & 2015 \\
\hline
\end{tabular}

\subsection{Estrutura do veículo}

A estrutura de um veículo é o principal componente do mesmo, por alocar todos os outros sistemas e subsistemas e ainda ter papal fundamental para manter o seu piloto em segurança. Com o objetivo básico de diminuição de consumo e consequentemente a redução na emissão de poluentes, deve-se buscar a redução do peso de todos os componentes do projeto. Desta forma, para o chassi do veículo foram realizadas simulações computacionais do chassi utilizando o software ANSYS nos auxiliando quanto ao dimensionamento, e a escola do melhor tipo de material para a estrutura do protótipo e, também, a disposição de todos os componentes a fim aproveitar todos os espaços.

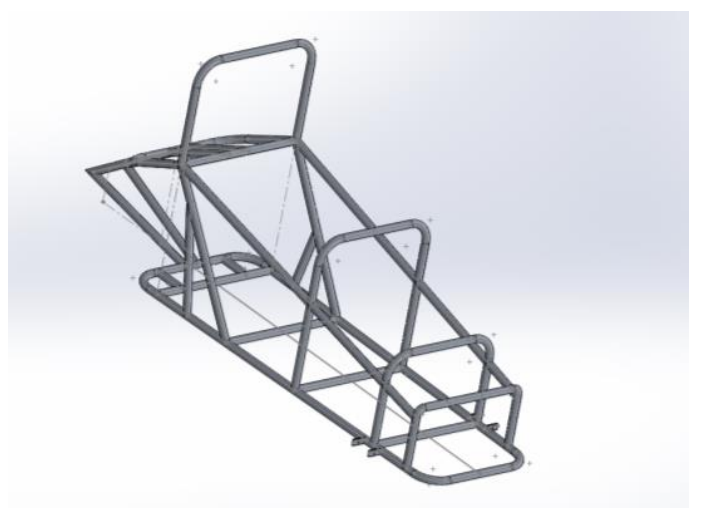

Figura 1: estrutura modelada em CAD (SolidWorks 2015) do veículo desenvolvido.

Ao finalizar a fase de modelagem do veículo, iniciou-se os diferentes tipos de simulações necessárias para o entendimento do comportamento da estrutura, quando submetida a vários tipos de cargas, e assim, se necessário intervir no modelo, a fim de solucionar possíveis problemas observados durante as simulações.

\subsection{Sistema de direção}

Para manter o veículo sobre o controle do piloto, será construído um sistema de direção baseado na geometria Ackerman. Quando um veículo realiza uma curva as rodas diretrizes tendem a realizarem circunferências diferentes, fazendo com que haja o escorregamento de uma das rodas. Essa geometria aplica um ângulo inferior a roda interior a curva e um ângulo mais acentuado a roda exterior a curva, fazendo com que as duas rodas sigam a 
trajetória de uma circunferência de mesmo centro, permitindo uma maior estabilidade e menor desgaste dos pneus.

Para determinação do ângulo mínimo da direção, deve-se estabelecer o ângulo mínimo de giro, desta forma determinou-se que o veículo realize curvas com 8 metros de raio, e suas medidas de entre eixos deve obrigatoriamente superior a $1000 \mathrm{~mm}$, e bitola com medida superior a 500 .

Através do estabelecimento destes critérios, é possível realizar o cálculo do ângulomínimo de direção necessário para a realização das curvas com segurança. Podemos estabelecer o ângulo mínimo através da fórmula (1):

$$
\text { Raio de curvatura }=\left(\frac{(t)}{2}\right)+\left(\frac{(l)}{\operatorname{sen}(A)}\right)
$$

Onde:

$t=$ Bitola do veículo

$l=$ Entre-eixo do veículo

$A=$ Ângulo de curvatura mínimo.

Substituindo-se os valores em (1) temos:

$$
A=\operatorname{sen}^{-1}+\left(\frac{1000 \mathrm{~mm}}{8000-\left(\frac{500}{2}\right)}\right)
$$

$$
A=7,415 \text { graus }
$$

Após a o desenho do protótipo em CAD, e a montagem do chassis e mangas de eixo, foi possível determinar as medidas base para o cálculo do ângulo de direção $\delta i$. Podemos observar os valores obtidos através da Tabela 2:

Tabela 2: dados do protótipo

\begin{tabular}{|c|c|}
\hline Ângulo de curvatura mínimo & 7,415 graus \\
\hline Bitola dianteira & $1045 \mathrm{~mm}$ \\
\hline Entre eixos & $1650 \mathrm{~mm}$ \\
\hline
\end{tabular}

Logo com todos os dados necessários, podemos calcular o ângulo $\delta_{i}$ das rodas internas a curva, através da fórmula (2):

$$
\operatorname{cotg} \delta_{0}-\operatorname{cotg} \delta_{i}=\frac{(t)}{(l)}
$$

Onde:

$t=$ Bitola do veículo.

$l=$ Entre-eixo do veículo.

$\delta_{0}=$ Ângulo de curvatura da roda externa a curva.

$\delta_{i}=$ Ângulo de curvatura da roda interna a curva. 
Adimitindo-se que $\mathrm{A}=\delta_{0}$, temos:

$$
\begin{gathered}
\delta_{i}=\operatorname{cotg}^{-1}\left[\operatorname{cotg} 7,415-\frac{1045}{1650}\right] \\
\delta_{i}=8,01 \text { graus }
\end{gathered}
$$

Com os ângulos de curvatura das rodas externa e interna determinados, podemos calcular o valor do ângulo de curvatura médio através da equação (5):

$$
\begin{gathered}
\operatorname{cotg} \delta=\frac{\operatorname{cotg} \delta_{0}+\operatorname{cotg} \delta_{i}}{2} \\
\operatorname{cotg} \delta=\frac{\operatorname{cotg} 7,415+\operatorname{cotg} 8,01}{2} \\
\delta=7,70 \text { graus }
\end{gathered}
$$

Finalizado os cálculos dimensionais, iniciou-se o processo de fabricação dos componentes da direção, utilizando um tubo de aço SAE 1020 com $22 \mathrm{~mm}$ diâmetro para a confecção da coluna de direção, terminais de rótula com M8 x 1,25, chapas de aço SAE 1020 para as conexões da barra de direção e fusos $8 \mathrm{~mm}$ utilizados como barra de direção.

\subsection{Frenagem do veículo}

O sistema de freio é responsável pela redução de velocidade do veículo, ou sua completa desaceleração. No protótipo a sistema escolhido utiliza discos e pinças hidráulicas de freio, por sua melhor eficiência na frenagem, frente aos sistemas convencionais (tambor de freio ou por sapatas), além de uma melhor dissipação de calor. O sistema é completamente adaptado de bicicletas, pois pelo veículo ser de pequeno porte e possuir um baixo peso, atende as necessidades do projeto.

Desta forma no projeto foram utilizados nas três rodas sistemas de freio a disco. Na dianteira foram instalados freios hidráulicos por possuírem maior facilidade de adaptação no acionamento e maior força aplicada no disco; fatores determinantes para a escolha deste tipo de freio, uma vez que, durante as frenagens ou curvas, os eixos dianteiros recebem o maio esforço devido a deslocamento do centro de massa do veículo. Já nos freios traseiros, são utilizados freios a disco convencionais (por cabos), acionados através de um pedal no interior do veículo. 




Figura 2: sistema de freios com as adaptações necessárias para a aplicação no projeto.

\subsection{Sistema de transmissão de potência}

O protótipo utiliza um câmbio automático do tipo CVT, já que este princípio de transmissão de potência é caracterizado pelo melhor aproveitamento de torque proveniente do motor através de suas infinitas relações de transmissão, demandando assim, de acordo com a rotação, mais torque para a retirada do veículo da inercia, ou mais velocidade às rodas, quando o veículo já se encontra em regime de potência (velocidades mais elevadas).

A caixa de câmbio escolhida na aplicação do projeto segue o conceito acima, e foi adequada de uma scooter $50 \mathrm{cc}$ (cilindrada), por possuir a rotação de acionamento semelhante ao motor utilizado no projeto, e ainda as relações de transmissão necessária, distribuindo o toque necessário para as rodas traseiras do protótipo, de acordo com a rotação do propulsor.

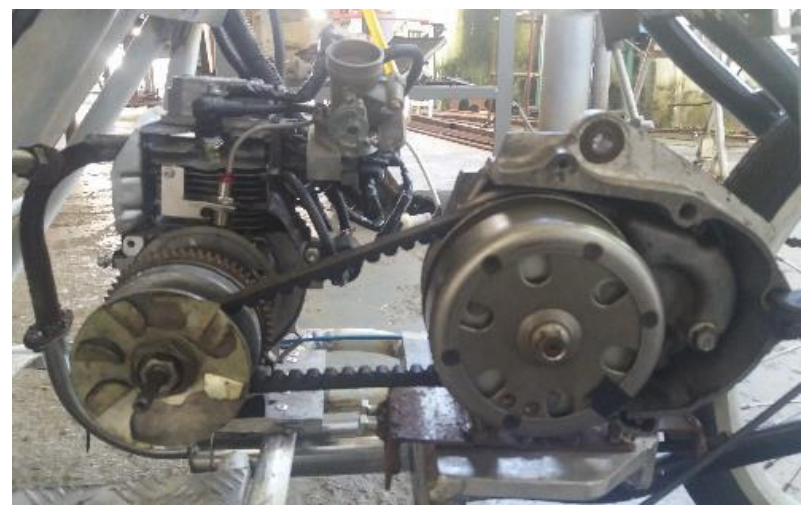

Figura 3: caixa de transmissão CVT adaptada ao protótipo.

\subsection{Sistema de injeção eletrônica}

Segundo Martins K.C.R [4] o módulo de injeção é o componente mais importante do sistema de injeção eletrônica, pois recebe todas as informações enviadas pelos sensores, analisando-as e comprando-as com os parâmetros salvos em sua memória; possibilitando assim comandar os atuadores, mantendo o funcionamento do motor sob qualquer condição de trabalho com a melhor eficiência possível. A ECU utilizada no projeto é similar aos utilizados em motocicletas de baixa cilindrada, permitindo a programação em tempo real através do software EcoCal da Ecotrons, possibilitando assim a alteração de todos os mapas de injeção e ignição conforme a necessidade do motor. Os sensores e atuadores 
utilizados possuem configuração semelhante ao já utilizados no mercado, com exceção do bico injetor, pois este é projetado com vazão mássica de combustível muito próximo ao ideal para o motor utilizado.

Para dar prosseguimento a este projeto, foi escolhido um bico injetor já disponível no mercado, já que o foco principal é a implementação de um sistema de injeção eletrônica, não sendo necessário considerar o projeto de um eletroinjetor. Desta forma, foi escolhido o bico fornecido pela Econtrons, por possuir características mais próximas ao demandado pelo projeto.

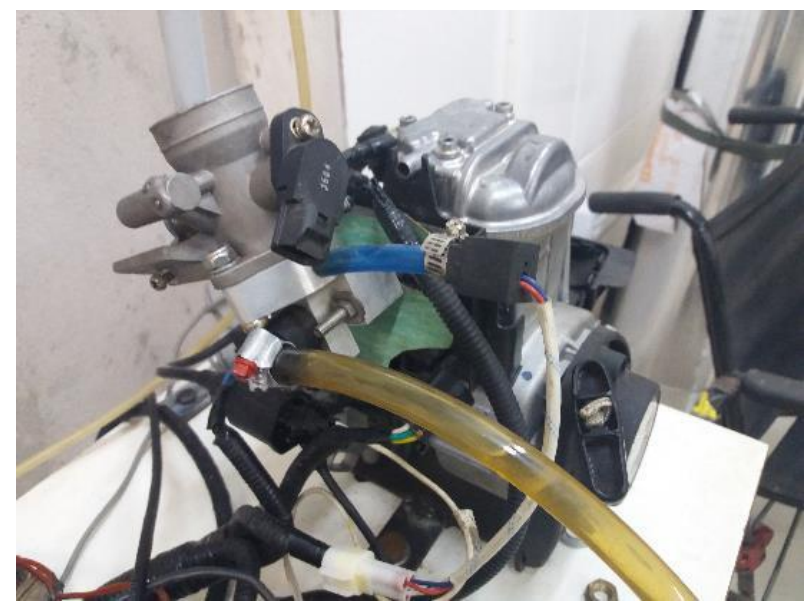

Figura 4: sistema de injeção eletrônica aplicado ao protótipo veicular.

\subsection{Análise estrutural do veículo}

Para a avaliação da rigidez e resistência mecânica da estrutura do veículo foram utilizadas análises computacionais com base no método de elementos finitos (MEF). Na análise estática da estrutura deve-se considerar esforções arbitrários, desprezando-se os efeitos das forças de inércias e amortecimento. Para a execução da simulação foram determinados como pontos de apoio, os suportes das rodas do veículo (fixações das mangas de eixo e quadro traseiro). Levou-se em conta ainda a massa estimada dos principais subsistemas do veículo (Tabela 3). Através desta análise é possível quantificar a deformação máxima dos membros da estrutura.

Tabela 3: massas estimadas a serem inseridas sobre o chassi do veículo.

\begin{tabular}{|c|c|}
\hline Sistema & Massa estimada \\
\hline Motor Honda GX 35 & $4 \mathrm{~kg}$ \\
\hline Sistema de transmissão CVT & $5,5 \mathrm{~kg}$ \\
\hline Sistema de direção & $3 \mathrm{~kg}$ \\
\hline Sistema de freio & $1,5 \mathrm{~kg}$ \\
\hline Carroceria & $10 \mathrm{~kg}$ \\
\hline Massa do piloto & $80 \mathrm{~kg}$ \\
\hline
\end{tabular}


Através desta análise podemos concluir que o chassi proposto apresenta a rigidez necessária, pois com a força aplicada a deformação foi ínfima, de aproximadamente 0,0009 m, no ponto máximo de esforço (partes em vermelho na Figura 5).

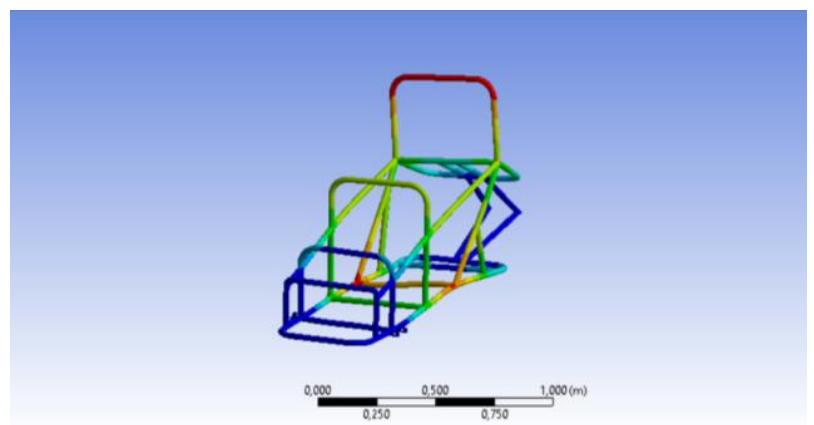

Figura 5: análise das deformações no chassi após a análise estrutural.

\subsection{Montagem final do protótipo}

Após o dimensionamento de todos os sistemas e subsistemas do veículo, iniciou-se o processo de montagem do protótipo, com a finalidade de executar os primeiros testes dinâmicos, avaliando o comportamento inicial, e efetuando possíveis ajustes decorrentes dos sistemas de direção, transmissão e freios (ajustes relacionados principalmente a alinhamentos de elementos flexíveis como correia e corrente de transmissão, folgas de parafusos, vibrações, entre outros.

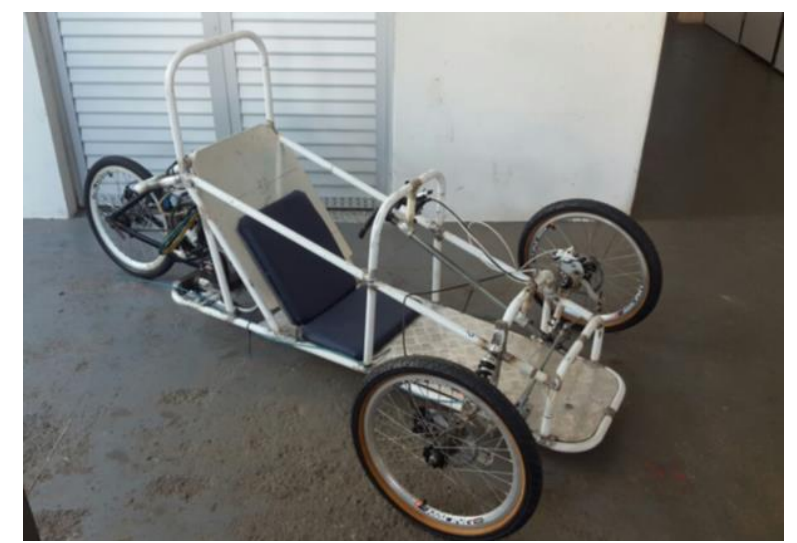

Figura 6: montagem do protótipo veicular.



Figura 7: carroceria provisória do protótipo veicular. 


\section{CONCLUSÃO}

O desenvolvimento do projeto aliou novas tecnologias e aprimoramentos nos sistemas que compõem um veículo automotor como: sistema de transmissão, direção e sistema de alimentação de combustível. Além disto, foi de importante valia na aplicação de conceitos aprendidos em sala de aula.

Podemos concluir, de acordo com os objetivos deste trabalho que:

- O chassi projetado através do software Solidworks e analisado através do software ANSYS, mostrou-se adequado para o desenvolvimento do protótipo, uma vez que, apresentou a resistência mecânica necessária, aliando-se ainda ao baixo peso, proporcionado pelo uso de uma liga de alumínio para a sua confecção;

- $\quad$ O sistema de direção dimensionado, apesar de possui uma concepção simples, atendeu as necessidades no que tange ao raio de curvatura, pois através de ajustes proporcionado pelo uso de diferentes ângulos Ackerman, possibilitou um rio de curvatura máximo com cerca de 9 metros;

- $\quad$ O sistema de transmissão mostrou-se eficiente quanto ao aproveitamento de torque proveniente do propulsor, pois possui uma variabilidade infinita de relações de transmissão;

- $\quad$ Por possuir um baixo peso, o sistema de freio mostrou-se adequado ao propósito do veículo;

\section{AGRADECIMENTOS}

Venho por meio deste agradecer o apoio do Instituto Federal de Educação, Ciência e Tecnologia do Maranhão - Campus Monte Castelo, por ter financiado e apoiado o presente projeto através do Programa Institucional de Bolsas de Iniciação em Desenvolvimento Tecnológico e Inovação (PIBITI); aos professores Keyll Carlos Ribeiro Martins e Evandro Martins Araújo Filho do Departamento de Mecânica e Materiais (DMM) do IFMA, e a todos meus colegas do Laboratório de Mecânica Automotiva pela ajuda em todo o desenvolvimento do projeto.

\section{REFERÊNCIAS}

[1] HEYWOOD, John, B. Internal combustion engine fundamentals. Nova York: McGraw Hill, 1988.

[2] PASSARINI, L. C. Análise e projeto de válvulas injetoras.1.ed. São Carlos, SP, 2002. EESC/USP.

[3] CASTRO, M. S. Uma metodologia para melhoria da rigidez torcional de componentes estruturais automotivos. Dissertação para obtenção de grau de mestre, UFSC, 2008.

[4] MARTINS, K.C.R; OLIVEIRA, G.H.S. Desenvolvimento e análise experimental de um sistema de injeção eletrônica de combustível em motores de Ciclo-Otto de pequeno porte. Projeto de iniciação científica - PIBITI. IFMA, São Luís, 2014.

[5] GILLISPIE, T. D. Fundamentals of Vehicle Dynamics. Society of Automotive Engineers Inc., EUA, 1992. 